\title{
Predictors of Cognitive Improvement during 12 Weeks of Antidepressant Treatment in Patients with Major Depressive Disorder
}

\author{
Jeong-Ok Lee', Ju-Wan Kim², Hee-Ju Kang ${ }^{2}$, Jin-Pyo Hong ${ }^{3}$, Jae-Min Kim² \\ 1 Department of Psychiatry, Naju National Hospital, Naju, ${ }^{2}$ Department of Psychiatry, Chonnam National University Medical School, Gwangju, \\ ${ }^{3}$ Department of Psychiatry, Sungkyunkwan University School of Medicine, Seoul, Korea
}

\begin{abstract}
Objective: Cognitive disturbance is one of the major symptoms of depression and may be improved by treatment with antidepressants. This study aimed to investigate the predictors of cognitive improvement in patients with major depressive disorder (MDD) who were taking antidepressants.

Methods: This study included 86 patients with MDD who completed 12 weeks of antidepressant monotherapy. Cognitive symptoms were assessed using the Perceived Deficits Questionnaire-Korean version (PDQ-K), which addresses four domains of cognitive functioning (attention/concentration, retrospective memory, prospective memory, and organization/ planning) and was administered at study entry and at the 12-week end point. A variety of demographic, clinical, and treatment-related variables were evaluated as predictors of changes in total and domain scores.

Results: All PDQ-K domains showed significant improvement after 12 weeks of antidepressant treatment. More severe initial depressive symptoms, fewer sick-leave days at study entry, and reduced use of concomitant anxiolytics/hypnotics during treatment were significantly associated with greater cognitive improvement.

Conclusion: Cognitive symptoms are more responsive to antidepressant treatment in patients with severe MDD. Reduced use of anxiolytics and hypnotics could improve the cognitive functioning of patients with MDD taking antidepressants.
\end{abstract}

KEY WORDS: Depression; Cognition; Antidepressive agents; Prediction; Anti-anxiety agents.

\section{INTRODUCTION}

Depression is a common mental disorder and one of the leading causes of morbidity and disability worldwide. ${ }^{1,2)}$ Depression is also associated with a significant economic burden due to missed work and reduced productivity. ${ }^{3)}$ The diagnostic criteria for major depressive disorder (MDD) in the Diagnostic and Statistical Manual of Mental Disorders, Fifth Edition (DSM-5) ${ }^{4)}$ include the presence of cognitive symptoms. Cognitive dysfunction has been associated with non-remission or relapse of depressive episodes ${ }^{5)}$ and impaired functioning and work

\footnotetext{
Received: February 1, 2018 / Revised: March 18, 2018 Accepted: May 28, 2018

Address for correspondence: Jae-Min Kim, MD, PhD Department of Psychiatry, Chonnam National University Medical School, 160 Baekseo-ro, Dong-gu, Gwangju 61469, Korea Tel: +82-62-220-6143, Fax: +82-62-225-2351

E-mail: jmkim@chonnam.ac.kr

ORCID: https://orcid.org/0000-0001-7409-6306
}

productivity. ${ }^{6-8)}$ Thus, one important goal of treatment for depression is improvement of cognitive functioning, ${ }^{9)}$ and the accurate evaluation of cognitive deficits is the first step toward achieving this goal.

Many studies have reported a positive effect of various antidepressants on cognition. ${ }^{10,11)}$ Recent comprehensive reviews confirmed that some cognitive symptoms can be improved by antidepressant treatment. ${ }^{12,13)}$ Numerous studies have reported on the correlates of cognitive function in patients with MDD. For example, a cross-sectional study found that premorbid intelligence and factors related to disease burden were independently associated with cognitive dysfunction in MDD. ${ }^{14)}$ However, little is known about the prospective predictors of cognitive improvement in patients with MDD taking antidepressants. A longitudinal study reported that clinical and sociodemographic factors were not associated with cognitive impairment. ${ }^{15)}$ The same study also suggested that neuro-

(c) This is an Open-Access article distributed under the terms of the Creative Commons Attribution Non-Commercial License (http://creativecommons.org/licenses/by-nc/4.0) which permits unrestricted non-commercial use, distribution, and reproduction in any medium, provided the original work is properly cited. 
psychological factors, such as processing speed impairment at baseline, were significant predictors of long-term global neurocognitive functioning. However, few clinical or treatment-related variables were examined, and the study was performed with a limited number of subjects.

Cognitive symptoms can be evaluated based on objective neuropsychological tests administered by experienced staff, or on the subjective reports of patients. Many neuropsychological tests have been validated for use with depressed patients, but these are of limited utility in a clinical situation because they are too time-intensive for application to a busy real-world practice and require an expert to administer them. ${ }^{16)}$ Subjective cognitive measures may overcome these limitations because they require less time and can be easily completed by self-report. Moreover, these measures can assess cognitive symptoms related to the individual perceptions and experiences of patients in their daily lives. To date, as far as we know, there has been no research evaluating the factors associated with the subjective cognitive changes experienced by patients with MDD during antidepressant treatment. Thus, this study aimed to investigate the predictors of cognitive improvement in patients with MDD taking antidepressants using a subjective cognitive measure. The early identification of factors that predict the severity of the illness could contribute to decisions about the strategies used to improve the cognitive function of patients with MDD.

\section{METHODS}

\section{Study Participants}

Patients with MDD were recruited from two university hospitals (Chonnam National University Hospital and Asan Medical Center) in South Korea from October 2013 to December 2014. The inclusion criteria for participation in this study were as follows: i) current diagnosis of a major depressive episode according to the DSM- 5 and confirmed with the Mini-International Neuropsychiatric Interview (MINI),17) ii) status as an outpatient of either sex aged 19 to 65 years, and iii) starting antidepressant monotherapy as either a new treatment or after changing from a previous monotherapy. The exclusion criteria were as follows: i) diagnosis of schizophrenia or other psychotic disorder, bipolar disorder, substance dependence, dementia, or other neurodegenerative disease that could sig- nificantly impact cognitive functioning, or a mood disorder due to a general medical condition or substance use; ii) pregnant or breastfeeding women or women who were less than 6 months post-partum; iii) inability to read or understand the information sheet, informed consent form, or patient-report questionnaires; iv) concurrent participation in a clinical trial; and/or v) acute suicidality based on the judgment of a clinician. In total, 86 patients completed both the 12-week antidepressant monotherapy treatment and the study. This study was approved by the Institutional Review boards of Chonnam National University Hospital and Asan Medical Center $(\mathrm{CNUH}-$ 2014-035), and all participants provided written informed consent.

\section{Cognitive Symptoms}

Cognitive symptoms were evaluated using the formally validated Perceived Deficits Questionnaire-Korean version (PDQ-K). ${ }^{18,19)}$ The PDQ-K consists of 20 items that assess four domains of cognitive functioning: attention/concentration, retrospective memory, prospective memory, and organization/planning. The PDQ-K is a self-administered questionnaire, and respondents rate the frequency of each complaint on a five-point Likert scale anchored from never (0) to almost always (4). The maximum score for each domain is 20 , with higher scores indicating more severe cognitive dysfunction. This study used the total score and the four domain scores for the analyses. Previous studies reported that the PDQ scores were significantly correlated with scores of several neuropsychological tests including Digit Symbol Substitution Test and Rey Auditory Verbal Learning Test. ${ }^{20}$

\section{Demographic Characteristics}

The demographic characteristics of patients were assessed at study entry by a clinical research coordinator using a structured case report form (CRF). Data were obtained regarding age, gender, duration of formal education, and current occupational status (currently employed or not).

\section{Clinical Characteristics}

At study entry, the clinical research coordinators assessed all other clinical characteristics, including history of depressive episodes (recurrent or first episode), using the structured CRF. Information regarding concurrent 
chronic medical disorders was gathered using a questionnaire that addressed six disorders, and these data were recoded as the presence or absence of any medical disorder. Depression severity was measured using the Montgomery - Asberg Depression Rating Scale (MADRS), ${ }^{21)}$ which consists of 10 items that yield a total score ranging from 0 to 60 . Disability was assessed using the Sheehan Disability Scale (SDS), ${ }^{22)}$ which measures disability at work, in social relationships, and in family life and yields a total score ranging from 0 to 30 . Quality of life was measured using the EuroQol-5 dimensions questionnaire (EQ-5D) health status thermometer, ${ }^{23)}$ which yields scores ranging from 0 to 100 . Sick-leave days (missed school or work) in the previous week due to depressive symptoms were also estimated. Higher scores on the MADRS and SDS, lower scores on the EQ-5D, and more sick-leave days were considered to indicate more severe symptoms and a greater impact of MDD.

\section{Treatment-related Characteristics}

The antidepressant choice for each patient was determined by their respective clinicians. The antidepressant types were divided into three categories: i) selective serotonin reuptake inhibitors (SSRIs), including citalopram, escitalopram, fluoxetine, paroxetine, and sertraline; ii) newer antidepressants, such as bupropion, venlafaxine, and mirtazapine; and iii) older antidepressants, such as amitriptyline, clomipramine, imipramine, milnacipran, nortriptyline, tianeptine, and trazodone. The use of concomitant medications, such as anxiolytics and/or hypnotics, that were not another type of antidepressant or an antipsychotic was allowed. All adverse events reported by the patients during the study period were recorded by the clinical research coordinators.

\section{Statistical Analyses}

The descriptive data obtained at the study entry are presented as the means \pm standard deviations (SD) or as percentages, as appropriate. The PDQ-K scores at study entry and at the 12-week end point were compared using paired $t$ tests. Differences in PDQ-K scores (12-week scores minus study-entry scores) were used as the dependent variables in the later analyses. Of the continuous independent variables, the MADRS has a previously validated cut-off point: $\leq 25$ for remission or mild depression vs. $\geq 26$ for moderate or more severe depression. ${ }^{24)}$ The other continuous independent variables have no accepted cut-off points and were therefore dichotomized by the median value for the analyses: age, $\leq 49$ vs. $\geq 50$ years; duration of education, $\leq 12$ vs. $\geq 13$ years; SDS, score of $\leq 21$ vs. $\geq 22$; EQ-5D, score of $\leq 49$ vs. $\geq 50$; and sick leave, $\leq 1$ vs. $\geq 2$ days in the previous week. These categorizations were used to facilitate the identification of patient characteristics in the univariate analyses. However, they were treated as continuous variables in the multivariate analyses to increase statistical power. In terms of type of antidepressant, older antidepressants were used by only three patients and were therefore excluded from the analysis. The univariate analyses compared differences in total and domain-specific PDQ-K scores according to the independent demographic, clinical, and treatment-related variables using $t$ tests. In addition, Pearson's correlation tests were carried out between the PDQ-K scores and independent continuous variables (i.e., scores on the MADRS, SDS, EQ-5D, and sick leave days) to recheck the associations between them and to maximize the statistical power. Variables showing significant associations in the univariate analyses $(p<0.05)$ were entered into the multivariate linear regressions to identify the independent predictors of cognitive improvement in total and domain-specific PDQ-K scores. All statistical analyses were performed using PASW Statistics ver. 18.0 software (IBM Co., Armonk, NY, USA).

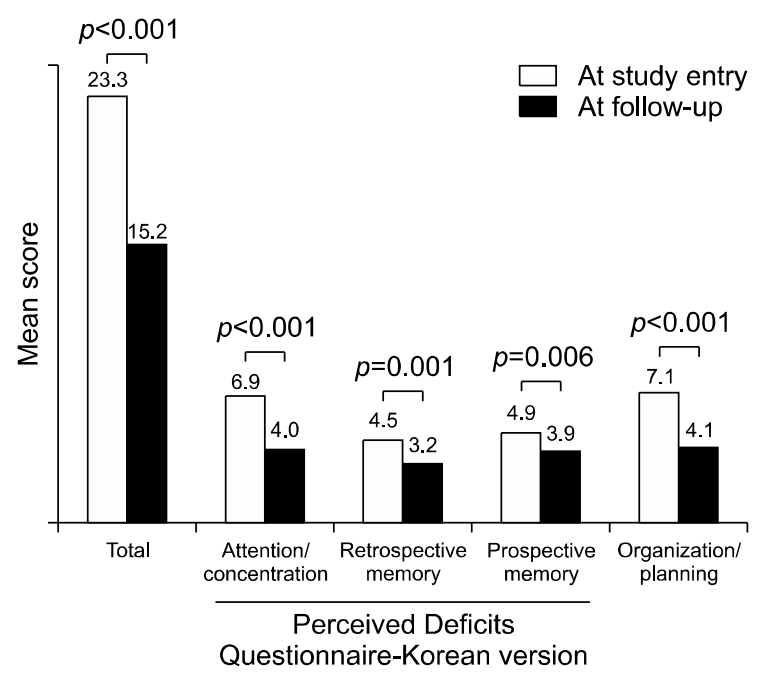

Fig. 1. Perceived Deficits Questionnaire-Korean version (PDQ-K) scores at study entry and after 12 weeks of treatment. 


\section{RESULTS}

\section{Patient Sample and Characteristics}

The mean (SD) age of the total sample was 48.8 (11.1) years, the number of women was $60(69.8 \%)$, the mean
(SD) duration of formal education was 10.9 (4.2) years, and $32(37.2 \%)$ participants were currently employed. Recurrent depression and chronic medical disorders were present in $20(23.3 \%)$ and $50(58.1 \%)$ patients, respectively. The mean MADRS score (SD) was 28.6 (7.2),

Table 1. Univariate associations between independent variables and changes in Perceived Deficits Questionnaire-Korean version (PDQ-K) scores

\begin{tabular}{|c|c|c|c|c|c|c|}
\hline Characteristic & Number & Total & $\begin{array}{c}\text { Attention/ } \\
\text { concentration }\end{array}$ & $\begin{array}{c}\text { Retrospective } \\
\text { memory }\end{array}$ & $\begin{array}{l}\text { Prospective } \\
\text { memory }\end{array}$ & $\begin{array}{l}\text { Organization/ } \\
\text { planning }\end{array}$ \\
\hline \multicolumn{7}{|l|}{ Demographic } \\
\hline \multicolumn{7}{|l|}{ Age $(y r)$} \\
\hline$<50$ & 37 & $-8.4(16.2)$ & $-3.0(4.9)$ & $-1.5(4.0)$ & $-0.8(3.7)$ & $-3.1(6.1)$ \\
\hline$\geq 50$ & 49 & $-7.9(11.9)$ & $-2.8(4.3)$ & $-1.1(3.2)$ & $-1.1(2.7)$ & $-2.9(4.4)$ \\
\hline \multicolumn{7}{|l|}{ Gender } \\
\hline Men & 26 & $-7.4(12.4)$ & $-2.5(3.8)$ & $-1.3(4.3)$ & $-1.2(2.9)$ & $-2.4(4.1)$ \\
\hline Women & 60 & $-8.4(14.5)$ & $-3.1(4.9)$ & $-1.2(3.2)$ & $-0.9(3.2)$ & $-3.2(5.6)$ \\
\hline \multicolumn{7}{|l|}{ Education (yr) } \\
\hline$<13$ & 59 & $-8.3(14.3)$ & $-2.9(4.7)$ & $-1.2(3.8)$ & $-1.2(3.2)$ & $-3.0(5.3)$ \\
\hline$\geq 13$ & 27 & $-7.9(13.0)$ & $-3.0(4.4)$ & $-1.4(3.1)$ & $-0.5(3.0)$ & $-3.0(5.0)$ \\
\hline \multicolumn{7}{|l|}{ Occupation } \\
\hline No & 54 & $-9.0(15.3)$ & $-3.2(5.1)$ & $-1.2(3.7)$ & $-1.2(3.1)$ & $-3.4(5.8)$ \\
\hline Have & 32 & $-6.7(10.9)$ & $-2.5(3.5)$ & $-1.3(3.4)$ & $-0.6(3.2)$ & $-2.3(3.8)$ \\
\hline \multicolumn{7}{|l|}{ Clinical } \\
\hline \multicolumn{7}{|l|}{ Recurrent depression } \\
\hline No & 66 & $-8.8(14.8)$ & $-3.3(4.8)$ & $-1.2(3.8)$ & $-1.0(3.3)$ & $-3.2(5.5)$ \\
\hline Yes & 20 & $-6.0(10.0)$ & $-1.8(3.4)$ & $-1.4(2.8)$ & $-0.7(2.4)$ & $-2.2(4.1)$ \\
\hline \multicolumn{7}{|c|}{ Chronic medical disorder } \\
\hline No & 36 & $-7.4(15.4)$ & $-2.4(4.2)$ & $-1.6(3.8)$ & $-0.6(3.5)$ & $-2.9(5.8)$ \\
\hline Have & 50 & $-8.6(12.7)$ & $-3.3(4.8)$ & $-1.0(3.4)$ & $-1.2(2.8)$ & $-3.1(4.7)$ \\
\hline \multicolumn{7}{|l|}{ MADRS (score) } \\
\hline$<26$ & 25 & $-4.2(10.5)$ & $-1.5(4.2)$ & $-0.9(3.0)$ & $-0.3(2.4)$ & $-1.2(3.93)$ \\
\hline$\geq 26$ & 61 & $-11.4(15.0)^{\dagger}$ & $-4.2(4.8)^{*}$ & $-1.7(4.3)$ & $-1.5(3.6)$ & $-4.5(5.7)^{\dagger}$ \\
\hline \multicolumn{7}{|l|}{ SDS (score) } \\
\hline$<22$ & 43 & $-5.7(10.3)$ & $-2.2(3.5)$ & $-0.8(3.0)$ & $-0.5(2.3)$ & $-2.2(3.6)$ \\
\hline$\geq 22$ & 43 & $-10.6(16.4)$ & $-3.7(5.3)$ & $-1.7(4.0)$ & $-1.4(3.7)$ & $-3.8(6.3)$ \\
\hline \multicolumn{7}{|c|}{ EQ-5D health status (score) } \\
\hline$<50$ & 34 & $-6.5(13.2)$ & $-2.5(4.1)$ & $-0.8(3.3)$ & $-0.3(3.1)$ & $-2.6(4.8)$ \\
\hline$\geq 50$ & 52 & $-10.7(14.5)$ & $-3.6(5.2)$ & $-1.9(3.9)$ & $-1.9(3.1)^{*}$ & $-3.5(5.8)$ \\
\hline \multicolumn{7}{|c|}{ Sick leave (days in the last week) } \\
\hline$<2$ & 43 & $-10.0(14.4)$ & $-3.3(4.8)$ & $-1.4(3.7)$ & $-1.6(3.3)$ & $-3.7(5.5)$ \\
\hline$\geq 2$ & 43 & $-6.3(13.1)$ & $-2.6(4.4)$ & $-1.1(3.4)$ & $-0.3(2.9)^{*}$ & $-2.3(4.8)$ \\
\hline \multicolumn{7}{|l|}{ Treatment related } \\
\hline \multicolumn{7}{|c|}{ Administered antidepressants } \\
\hline SSRIs & 48 & $-9.4(15.3)$ & $-2.9(4.9)$ & $-1.8(3.4)$ & $-1.3(3.7)$ & $-3.3(5.9)$ \\
\hline NADTs & 36 & $-6.6(12.1)$ & $-3.0(4.3)$ & $-0.7(3.6)$ & $-0.5(2.3)$ & $-2.5(4.3)$ \\
\hline \multicolumn{7}{|c|}{ Concomitant anxiolytics/hypnotics } \\
\hline No & 35 & $-10.2(14.2)$ & $-3.6(4.8)$ & $-1.6(3.8)$ & $-1.4(3.2)$ & $-3.7(5.3)$ \\
\hline Yes & 51 & $-5.1(12.9)^{*}$ & $-1.9(4.0)^{*}$ & $-0.8(3.2)$ & $-0.4(3.0)$ & $-1.9(4.9)^{*}$ \\
\hline \multicolumn{7}{|l|}{ Any side effects } \\
\hline No & 73 & $-10.8(14.3)$ & $-4.9(5.7)$ & $-1.5(3.4)$ & $-1.2(2.7)$ & $-3.2(5.2)$ \\
\hline Yes & 13 & $-7.7(13.8)$ & $-2.6(4.3)^{*}$ & $-1.2(3.6)$ & $-0.9(3.2)$ & $-2.9(5.2)$ \\
\hline
\end{tabular}

Values are presented as mean (standard deviation).

MADRS, Montgomery Asberg Depression Rating Scale; SDS, Sheehan Disability Scale; EQ-5D, EuroQol-5 dimensions questionnaire; SSRIs, selective serotonin reuptake inhibitors; NADTs, newer antidepressants.

${ }^{*} p<0.05,{ }^{\dagger} p<0.01$ by $t$ tests. 
the mean SDS score was 20.3 (8.0), and the mean EQ-5D score was 50.2 (18.4). The mean (SD) number of sick-leave days in the last week was 2.6 (2.9) days. SSRIs and newer antidepressants were taken by 48 (55.8\%) and $36(41.9 \%)$ patients, respectively. Concomitant anxiolytics/hypnotics were used by 51 (59.3\%) patients, and the rate of use did not differ significantly by MADRS score group ( $\leq 25,53.1 \% ; \geq 26,63.0 \% ; p=0.297$ ). Drug side effects were reported by 13 (15.1\%) patients.

\section{PDQ-K Score Changes}

Total and domain-specific changes in PDQ-K scores from study entry to the 12-week endpoint are presented in Figure 1. The PDQ-K total and domain scores were significantly decreased after 12 weeks of antidepressant treatment. The mean (SD) score changes were as follows: total score: -8.1 (13.8), attention/concentration domain; -2.9 (4.6), retrospective memory domain; -1.3 (3.5), prospective memory domain; $-1.0(3.1)$, and organization/planning domain; - 3.0 (5.2).

\section{Univariate Associations with PDQ-K Score Changes}

The univariate associations between independent variables and changes in PDQ-K scores are summarized in Table 1. The power estimates (calculated by an online program: http://www.dssresearch.com/KnowledgeCenter/ toolkitcalculators/statisticalpowercalculators.aspx) of changes in the PDQ-K score as a function of demographic characteristics was $72 \%$ to $78 \%$, being $84 \%$ to $93 \%$ for clinical characteristics and $75 \%$ to $90 \%$ for treatment-related characteristics. Greater decreases in PDQ-K total scores were significantly associated with higher MADRS scores and no concomitant use of anxiolytics/hypnotics. Greater decreases in PDQ-K attention/concentration scores were significantly associated with higher MADRS scores, no concomitant use of anxiolytics/hypnotics, and no drug side effects. Greater decreases in PDQ-K prospective memory scores were significantly associated with higher EQ-5D scores and fewer sick-leave days in the last week. Greater decreases in PDQ-K organization/planning scores were significantly associated with higher MADRS scores and no concomitant use of anxiolytics/hypnotics. No associations were found with regard to the retrospective memory domain scores. Duration of education was not associated with changes in PDQ-K scores, irrespective of whether the latter was treated as a discrete or continuous variable. The results (strengths of associations) on the Pearson's correlation tests between the PDQD-K scores and scores on the MADRS, SDS, EQ-5D, and sick leave days were not substantially different from the $t$ tests ones.

\section{Multivariate Associations with Score Changes on the PDQ-K}

The multivariate associations of independent variables with PDQ-K score changes are summarized in Table 2. Greater decreases in PDQ-K total and attention/concentration scores were independently associated with higher MADRS scores and no concomitant use of anxiolytics/ hypnotics. Additionally, greater decreases in PDQ-K prospective memory scores were independently associated with fewer sick-leave days in the last week, and greater decreases in PDQ-K organization/planning scores were independently associated with higher MADRS scores.

\section{DISCUSSION}

According to our results, the subjective cognitive symptoms of patients with MDD, as evaluated by the PDQ-K, were significantly improved after 12 weeks of antidepressant treatment. Moreover, such improvement was significantly predicted by depression severity, sick-leave

Table 2. Multivariate associations between independent variables and changes in Perceived Deficits Questionnaire-Korean version scores

\begin{tabular}{lccc}
\hline \multicolumn{1}{c}{ Variable } & Total & $\begin{array}{c}\text { Attention/ } \\
\text { concentration }\end{array}$ & $\begin{array}{c}\text { Prospective } \\
\text { memory }\end{array}$ \\
\hline MADRS, higher scores & $-0.5(-0.9 \text { to }-0.1)^{*}$ & $-0.3(-0.6 \text { to }-0.1)^{*}$ & $\begin{array}{c}\text { Organization/ } \\
\text { planning }\end{array}$ \\
EQ-5D health status, higher scores & - & - & $-0.1(-0.6$ to 0.3$)$ \\
Sick leave, less days in the last week & - & - & $-0.3(-0.6 \text { to }-0.1)^{*}$ \\
Concomitant anxiolytics/hypnotics, yes vs. no & $-5.4(-9.9 \text { to }-0.9)^{*}$ & $-2.7(-3.3 \text { to }-0.9)^{*}$ & - \\
Any side effects, no vs. yes & - & $-2.1(-4.5$ to 0.6$)$ & $-0.3)^{\dagger}$ \\
\hline
\end{tabular}

Values are presented as B (95\% confidence interval). 
days at study entry, and concomitant use of anxiolytics/hypnotics during the study period. These findings show that the subjective cognitive appraisal of patients with MDD is improved by the use of antidepressants, which is partly consistent with previous studies using objective neuropsychological tests to assess cognitive function. ${ }^{10,11)}$ To our knowledge, this is the first study to evaluate subjective cognitive symptoms using a self-administered questionnaire completed by patients with MDD. Overall, the results of the previous and current studies suggest that both the objective and the subjective cognitive symptoms of patients with MDD can be improved by antidepressant treatment.

PDQ-K total, attention/concentration, and organization/planning scores were significantly more improved in patients with more severe MDD at study entry. This suggests that the cognitive disturbance was significantly associated with the general depressive pathology ${ }^{25)}$ and that treatment is more effective for severe pathology; it also suggests that improved cognitive functioning is not an aspect of remission. That is, the same rate of improvement in the symptoms of patients with more and less severe MDD can be interpreted as reflective of greater improvement in the former group; however, these findings should be interpreted cautiously. The results also suggest that the decrease in cognitive functioning in severely depressed patients is not permanent.

Number of sick-leave days was significantly associated with less improvement in the PDQ-K prospective scores. Several previous studies showed that the severity of depressive symptoms influenced the level of success in returning to work, ${ }^{26,27)}$ with more severe depression increasing the number of sick-leave days taken. Our findings reflect the reverse direction, as more sick-leave days were associated with poorer cognitive functioning in patients with MDD. This was probably due to lack of physical activity, which is consistent with previous studies showing that physical activity positively influences cognitive functioning. ${ }^{28,29)}$

Concomitant use of anxiolytics and hypnotics was significantly associated with less improvement in subjective cognitive symptoms according to the PDQ-K total and attention/concentration scores. Previous studies have found that extensive and prolonged use of benzodiazepines produce cognitive impairment. ${ }^{30,31)}$ Our findings suggest that anxiolytics and hypnotics should be used only when ab- solutely necessary for improving the cognitive symptoms of patients with MDD. However, this finding should be treated cautiously, as a higher dose of anxiolytics might be prescribed for patients with more severe depressive symptoms. The doses of anxiolytics were not evaluated in this study, although there was no difference in the frequency of anxiolytic administration between the more and less severely depressed patients.

Finally, there was no significant relationship between subjective cognitive improvement and demographic characteristics, such as age, gender, years of formal education, or occupation. Additionally, the clinical characteristics of a recurrent depressive episode, the presence of a comorbid chronic medical disorder, and the SDS scores were not significantly related to cognitive improvement. Our findings agree with those of one previous study ${ }^{15)}$ that showed no significant relationship between cognitive impairment and demographic characteristics, such as age, gender, or years of education.

The strengths of this study include the use of a structured research protocol, well-recognized and standardized scales, and a prospective study design. The limitations of this study include the fact that the particular treatment modalities for each patient were determined by the clinicians rather than by any formal guidelines; therefore, inter-clinician variability may have affected the observed outcomes. Objective cognitive tests were not administered, although it has been reported that the correlations between the PDQ and neuropsychological tests were significant. ${ }^{20)}$ In addition, our study was designed to investigate the a priori predictors of subjective cognitive changes; indeed, this is the first study to use a subjective cognitive measure in this context. As with other novel findings, these results should be reexamined in a larger cohort of depressed patients, and should be compared with data obtained from objective neuropsychological tests of cognitive functioning. Although our sample was predominantly female $(69.8 \%)$, the gender ratio was similar to that of a previous, large-scale and nationally representative study of Koreans (women, $74 \%$ ). ${ }^{32)}$ Nevertheless, the sample size of our study was not sufficient, particularly for detecting changes in PDQ-K scores as a function of demographic characteristics. In relation to this, the associations between the PDQD-K scores and all independent variables were relatively weak in that all strengths of the associations lost significance if multiple 
corrections are administered. Future studies with larger sample would be anticipated.

Cognitive symptoms have recently been recognized as one of the primary outcomes of depression treatment, ${ }^{33)}$ and the effects of several antidepressants on the cognitive functioning of patients with MDD have been investigated. $^{20,34)}$ This study demonstrated that patients with severe MDD showed more improvement in their subjective cognitive functioning in response to antidepressant treatment. Therefore, active antidepressant pharmacotherapies can help to improve the subjective cognitive symptoms of patients with MDD. Efforts to encourage such patients to return to work may also improve their cognition by increasing their physical activity. Additionally, restricting the use of anxiolytics and hypnotics may improve the subjective cognitive functioning of patients with MDD who take antidepressants.

\section{Acknowledgments}

The study was supported by a grant of the Korea Health Technology R\&D Project through the Korea Health Industry Development Institute (KHIDI), funded by Ministry of Health \& Welfare, Republic of Korea (HC15C1405).

\section{REFERENCES}

1. Kessler RC, Berglund $\mathrm{P}$, Demler $\mathrm{O}$, Jin R, Koretz D, Merikangas KR, et al. The epidemiology of major depressive disorder: results from the National Comorbidity Survey Replication (NCS-R). Jama 2003;289:3095-3105.

2. Moussavi S, Chatterji S, Verdes E, Tandon A, Patel V, Ustun B. Depression, chronic diseases, and decrements in health: results from the world health surveys. Lancet 2007;370:851858.

3. Stewart WF, Ricci JA, Chee E, Hahn SR, Morganstein D. Cost of lost productive work time among US workers with depression. JAMA 2003;289:3135-3144.

4. American Psychiatry Association; American Psychiatric Association. DSM-5 Task Force. Diagnostic and statistical manual of mental disorders: DSM-5. 5th ed. Washington, DC:American Psychiatric Association;2013.

5. Judd LL, Akiskal HS, Maser JD, Zeller PJ, Endicott J, Coryell W, et al. Major depressive disorder: a prospective study of residual subthreshold depressive symptoms as predictor of rapid relapse. J Affect Disord 1998;50:97-108.

6. Jaeger J, Berns S, Uzelac S, Davis-Conway S. Neurocognitive deficits and disability in major depressive disorder. Psychiatry Res 2006; 145:39-48.

7. Woo YS, Rosenblat JD, Kakar R, Bahk WM, Mclntyre RS. Cognitive deficits as a mediator of poor occupational function in remitted major depressive disorder patients. Clin Psychopharmacol Neurosci 2016;14:1-16.

8. Kim HG, Kong EJ, Cheon EJ, Kim HW, Koo BH. Association between cerebral amyloid deposition and clinical factors including cognitive function in geriatric depression: pilot study using amyloid positron emission tomography. Clin Psychopharmacol Neurosci 2016;14:378-382.

9. Danner M, Hummel JM, Volz F, van Manen JG, Wiegard B, Dintsios CM, et al. Integrating patients' views into health technology assessment: analytic hierarchy process (AHP) as a method to elicit patient preferences. Int J Technol Assess Health Care 2011:27:369-375.

10. Soczynska JK, Ravindran LN, Styra R, McIntyre RS, Cyriac A, Manierka MS, et al. The effect of bupropion XL and escitalopram on memory and functional outcomes in adults with major depressive disorder: results from a randomized controlled trial. Psychiatry Res 2014;220:245-250.

11. Mahableshwarkar AR, Zajecka J, Jacobson W, Chen Y, Keefe RS. A randomized, placebo-controlled, active-reference, double-blind, flexible-dose study of the efficacy of vortioxetine on cognitive function in major depressive disorder. Neuropsychopharmacology 2015;40:2025-2037.

12. Rosenblat JD, Kakar R, Mclntyre RS. The cognitive effects of antidepressants in major depressive disorder: a systematic review and meta-analysis of randomized clinical trials. Int $)$ Neuropsychopharmacol 2015;19:pyv082.

13. Keefe RS, McClintock SM, Roth RM, Doraiswamy PM, Tiger S, Madhoo M. Cognitive effects of pharmacotherapy for major depressive disorder: a systematic review. J Clin Psychiatry 2014;75:864-876.

14. Elgamal S, Denburg S, Marriott M, MacQueen G. Clinical factors that predict cognitive function in patients with major depression. Can J Psychiatry 2010;55:653-661.

15. Gu CZ, He HL, Duan HF, Su ZH, Chen H, Gan JL. Predictors of neurocognitive impairment at 2years after a first-episode major depressive disorder. Compr Psychiatry 2016;68:24-33.

16. Evans VC, Iverson GL, Yatham LN, Lam RW. The relationship between neurocognitive and psychosocial functioning in major depressive disorder: a systematic review. J Clin Psychiatry 2014;75:1359-1370.

17. Sheehan DV, Lecrubier $Y$, Sheehan $\mathrm{KH}$, Amorim P, Janavs J, Weiller E, et al. The Mini-International Neuropsychiatric Interview (M.I.N.I.): the development and validation of a structured diagnostic psychiatric interview for DSM-IV and ICD-10. J Clin Psychiatry 1998;59 Suppl 20:22-33;quiz 34-57.

18. Kim JM, Hong JP, Kim SD, Kang HJ, Lee YS. Development of a Korean version of the Perceived Deficits QuestionnaireDepression for Patients with Major Depressive Disorder. Clin Psychopharmacol Neurosci 2016;14:26-32.

19. Takasaki H, Chien CW, Johnston V, Treleaven J, Jull G. Validity and reliability of the perceived deficit questionnaire to assess cognitive symptoms in people with chronic whip- 
lash-associated disorders. Arch Phys Med Rehabil 2012;93: 1774-1781.

20. McIntyre RS, Lophaven S, Olsen CK. A randomized, double-blind, placebo-controlled study of vortioxetine on cognitive function in depressed adults. Int J Neuropsychopharmacol 2014; 17:1557-1567.

21. Montgomery SA, Asberg M. A new depression scale designed to be sensitive to change. Br J Psychiatry 1979;134:382-389.

22. Leon AC, Olfson M, Portera L, Farber L, Sheehan DV. Assessing psychiatric impairment in primary care with the Sheehan disability scale. Int I Psychiatry Med 1997;27:93105.

23. EuroQol Group. EuroQol--a new facility for the measurement of health-related quality of life. Health Policy 1990;16: 199-208.

24. Bose A, Tsai J, Li D. Early non-response in patients with severe depression: escitalopram up-titration versus switch to duloxetine. Clin Drug Investig 2012;32:373-385.

25. McDermott LM, Ebmeier KP. A meta-analysis of depression severity and cognitive function. J Affect Disord 2009;119:1-8.

26. de Vries G, Koeter MW, Nabitz U, Hees HL, Schene AH. Return to work after sick leave due to depression; a conceptual analysis based on perspectives of patients, supervisors and occupational physicians. J Affect Disord 2012;136: 1017-1026.

27. Blank L, Peters J, Pickvance S, Wilford J, Macdonald E. A systematic review of the factors which predict return to work for people suffering episodes of poor mental health. I Occup
Rehabil 2008;18:27-34.

28. Cai H, Li G, Hua S, Liu Y, Chen L. Effect of exercise on cognitive function in chronic disease patients: a meta-analysis and systematic review of randomized controlled trials. Clin Interv Aging 2017;12:773-783.

29. Kramer AF, Erickson $\mathrm{KI}$, Colcombe SJ. Exercise, cognition, and the aging brain. J App/ Physiol (1985) 2006;101:1237- 1242.

30. Barker MJ, Greenwood KM, Jackson M, Crowe SF. Cognitive effects of long-term benzodiazepine use: a meta-analysis. CNS Drugs 2004; 18:37-48.

31. Verster JC, Volkerts ER, Verbaten MN. Effects of alprazolam on driving ability, memory functioning and psychomotor performance: a randomized, placebo-controlled study. Neuropsychopharmacology 2002;27:260-269.

32. Yang SJ, Kim SY, Stewart R, Kim JM, Shin IS, Jung SW, et al. Gender differences in 12-week antidepressant treatment outcomes for a naturalistic secondary care cohort: the CRESCEND study. Psychiatry Res 2011;189:82-90.

33. McIntyre RS. Using measurement strategies to identify and monitor residual symptoms. J Clin Psychiatry 2013;74 Suppl 2:14-8.

34. Raskin J, Wiltse CG, Siegal A, Sheikh J, Xu J, Dinkel J, et al. Efficacy of duloxetine on cognition, depression, and pain in elderly patients with major depressive disorder: an 8-week, double-blind, placebo-controlled trial. Am J Psychiatry 2007; 164:900-909. 